\title{
Hope in the hunt for brain disorder biomarkers
}

\author{
— Cite as: CMAJ 2017 January 30;189:E182-3. doi: 10.1503/cmaj.1095380
}

magine trying to fight a fire blindfolded. You can smell smoke, but what do you use to put out the blaze if you don't know how it's fueled?

Brain disorders pose a similar challenge in medicine. Despite progress in neuroimaging, clinicians and researchers still lack reliable biological measures, known as biomarkers, for complex disorders like autism, dementia and depression. Diagnosis is based on a patient's symptoms, the smoke to the disorder's unseen fire. This complicates treatment because people may suffer similar symptoms for different reasons, just as smoke can come from different types of fire, each requiring a specific extinguisher.

Researchers supported by the Ontario Brain Institute are starting to think outside the diagnostic box in their search for clues to the underlying causes of toughto-treat disorders. "We want to start looking across disorders at people who have similar types of symptoms," said Dr. Stephanie Ameis, a clinician-scientist at the Centre for Addiction and Mental Health (CAMH) in Toronto.

People who share a specific symptom may have more in common than those who share a diagnosis, Ameis explained at a presentation on the year ahead in brain research. Particularly when it comes to autism, patients with the same diagnosis may "vary hugely" in terms of symptoms and functioning, she said. "Even very promising treatments have failed, maybe because the [test] groups have been too diverse."

Researchers can tune out the noise by focusing on an "underlying group type" that cuts across diagnoses, Ameis said. For example, problems with social cognition are common in many patients with brain disorders. "If we look across these groups, maybe we'll understand how different degrees of social cognitive impairment imprint on a specific circuit," she said.
Using this approach, Ameis found that children with autism, attention deficit hyperactivity disorder and obsessive compulsive disorder have similar impairments in the wiring connecting the left and right sides of the brain. Her study, published in the American Journal of Psychiatry in July 2016, also linked greater impairment to greater challenges in everyday functioning, regardless of diagnosis.

"If we understand better what the similarities are, maybe we can use the same treatment across these kids," Ameis said.

Ameis is now studying how adults with autism, bipolar disorder and schizophrenia differ on measures of social cognition from healthy controls. Among the study's early findings, "individuals with bipolar disorder and healthy controls perform best on social cognitive tasks, like reading facial expressions," she said. Participants with schizophrenia performed worse on certain tasks, she added. "It seems to be associated with alterations in the structure of a particular circuit."

If researchers are able to pin down biomarkers that reliably indicate or predict brain disorders, they may open new avenues for treatment and prevention through the modification of those markers.

Dr. Sanjeev Kumar, a geriatric psychiatrist at $\mathrm{CAMH}$, is studying whether impairments in neuroplasticity, the brain's ability to restructure itself, could be both a marker and target for treatment of Alzheimer disease. He's using electroencephalogram recordings of the brain's electrical activity to measure how patients with Alzheimer disease respond to magnetic stimulation of brain regions that control cognitive function.

Transcranial magnetic stimulation has been used before to assess and enhance neuroplasticity in other areas of the brain, and the technique holds promise as a treatment for Alzheimer disease. "If we

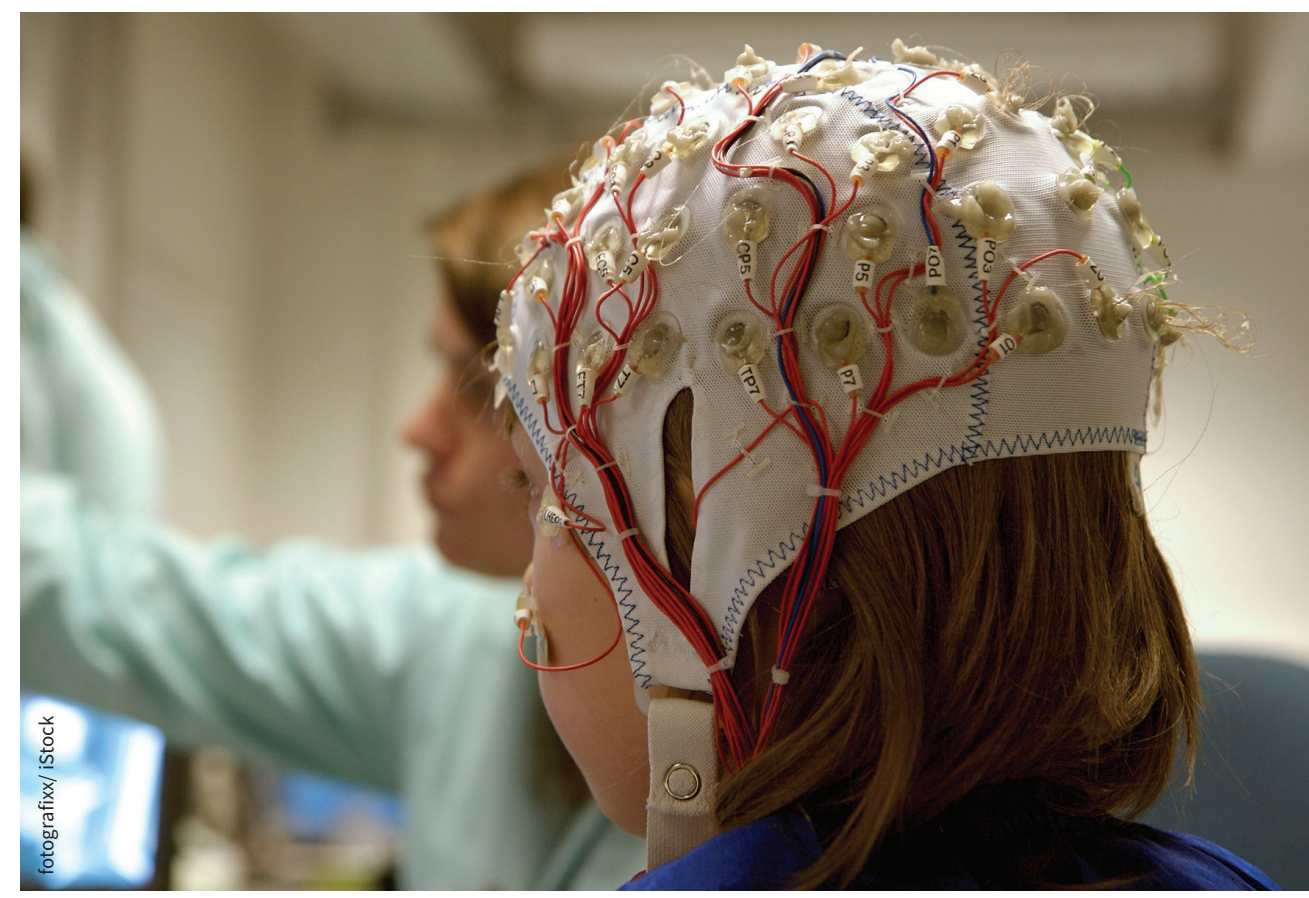

Ontario Brain Institute researchers are hot on the trail of better biological measures to help diagnose and treat brain disorders. 
can characterize the illness better in terms of neuroplasticity or other biomarkers combined with this intervention, then we'll be able to better titrate this for future use or rule it out," says Kumar.

In his study, patients with Alzheimer disease showed "clear differences" in neuroplasticity compared to healthy controls. Kumar will publish his findings later this year.

Other researchers are hoping to uncover biomarkers that could be used to predict risk for treatment-resistant depression and suicide.
About a third of people with depression don't respond to treatment, and among patients who are suicidal, only some attempt to kill themselves. "The big problem that we're facing is that we don't understand what's happening mechanistically in the brain in both of these cases," said Sakina Rizvi, a scientist at St. Michael's Hospital in Toronto.

People with treatment-resistant depression report higher levels of anhedonia, or loss of pleasure, even after controlling for depression severity. "Anhedonia is also linked with increased levels of suicidal ide- ation," noted Rizvi. "What's also interesting about this group is that they have high levels of psychological pain, but less response to physical pain, so if you touch them they won't feel that as much."

Rizvi suspects these patients have a shared impairment in an area of the brain where the wiring for reward, pain and attention overlap. She will be conducting brain scans to locate that impairment "with the hope we will be able to predict who is at risk for suicide attempt."

Lauren Vogel, CMAJ 